\title{
STUDY OF VARIATIONS IN E.C.G. IN NORMAL INDIVIDUALS.
}

M. Padma Geethanjali, M. Usha Rani.

1. Professor, Department of Physiology, Andhra Medical College, Visakhapatnam.

2. Associate Professor, Department of Physiology, Andhra Medical College, Visakhapatnam

\section{CORRESPONDING AUTHOR:}

Dr. M. Padma Geethanjali,

Professor, Department of Physiology,

Andhra Medical College,

Visakhapatnam-530002, A.P.

E-mail: gmpadma@yahoo.com

\begin{abstract}
The present study includes100 normal healthy individuals both males and females, 28 females and 72 males within the age groups from 16 years to 58 years were taken after careful clinical Examination to rule out any cardiac abnormality in particular, and other diseases in general; by following the given pattern. It is known that pulmonary, endocrinal, renal electrolytes and other abnormalities all can result in changes in the ECG pattern; a detailed careful clinical examination was done to rule out the above disorders. Infants were excluded from the study, as post partum period may be associated with changes pertaining to fetal circulation, which needs a separate study. Children below 15 years were also excluded from the study because incidence of congenital heart disease is more common in ages less than 15 years. Symptomatic individuals with any system were also not included in this study.

Present study aims at Studying the: Rate, Rhythm, Axis, PR Interval, QRS duration, QT duration, PR and ST segments in a systematic way to note the following: 1.To see any abnormalities in all above parameters. (a)To note their frequency; (b)Whether they are related to age; (c)The types of abnormalities, (d)And to note a particular abnormality, whether it is a normal variant or associated with a silent diseased state (e)To study the variance and distribution of all the parameters.
\end{abstract}

The 12 lead ECG(Electrocardiograph)Machine was taken for this study with $10 \mathrm{~mm}$ standardization. Resting ECG(Electrocardiogram) was taken for all the individuals. Conclusion: ECG varies from individual to individual even in normal individuals. Majority of the ECGs in normal individuals shows a pattern, which falls within normal range. A considerable percentage of individuals deviate from the normal pattern. The frequently observed abnormalities are Tachycardia, Bradycardia and ERS(Early Repolarisation Syndrome).Less frequently seen abnormalities are LAD(Left Axis Deviation), $1^{0}$ heart block PVCs(Premature Ventricular Contractions) wandering pacemaker, RBBB(Right Bundle Branch Block)So understanding normal ECG and deviations in normal individuals is very important to interpret the diseased states, treating them, and giving the prognosis.

KEY WORDS: ECG, Physiological variations, Normal individuals.

INTRODUCTION: Every contraction of the heart is preceded by electrical activity. This electrical activity in human being starts in S.A. Node and travels through specialized fibers spreading through 
the myocardium. The same electrical activity is passed to the surrounding tissues and finally to all parts of the body. Needless to say the intensity of electrical activity weakens as the distance increases from the heart. Hence the Electrical activity can be picked up from any part of the body. However it needs to be augmented while recording Example AVR, AVL, AVF.

Routine ECG is recorded from standard points of the body - limbs and chest. Study of ECG includes the intervals, axes of various waves, wave patterns. These parameters do change from individual to individual but lying in a range. However, there are some deviations either due to disease or in normal individual also. The present study aims at knowing the variations in normal individuals.

METHODS AND MATERIALS:100 normal healthy individuals both males and females, 28 females and 72 males within the age groups from 16 years to 58 years were taken after careful clinical Examination to rule out any cardiac abnormality in particular, and other diseases in general; by following the given pattern. It is known that pulmonary, endocrinal, renal electrolytes and other abnormalities, all can result in changes in the ECG pattern, a detailed careful clinical examination was done to rule out the above disorders. Infants were excluded from the study, as post partum period may be associated with changes pertaining to fetal circulation, which needs a separate study. Children below 15 years were also excluded from the study because incidence of congenital heart disease is more common in ages less than 15 years which show abnormalities in ECG. Symptomatic individuals with any system were not included.

Only those individuals with all normal parameters were included in this study. A resting 12 lead ECG was taken in all individuals with $10 \mathrm{~mm}$ standardization. The ECG in the following leads $\mathrm{L} \mathrm{I}, \mathrm{L}$ II, L III, AVR, AVL, AVF, $V_{1} V_{2} V_{3} V_{4} V_{5} V_{6}$ were taken.

ELECTRO CARDIO GRAPHIC LEADS: To record in ECG an electric circuit between the heart and the electrocardiograph must be completed. For this purpose electrodes are placed on different parts of the body surface and are connected to the instrument by means of cables. Thus the whole system consists of an instrument, electrodes, cables and leads.

STANDARD LEADS: The standard leads are called bipolar leads because they are composed of two electrodes - one that is negative and one that is positive- and the ECG records the difference in electrical potential between them.

Lead I: is composed of right arm, which is designated as negative and the left arm, which is considered positive.

Lead II: is composed of right arm which is made negative and the left leg, which is considered positive.

Lead III: is made up of the left arm which is designated as negative and the left leg which is considered positive.

The three standard leads form a triangle over the body and have a mathematical relationship to one another as described by Einthoven. The height or depth of the recordings in Lead I and Lead III equals the height (or) depth of the recordings in Lead II. 
Lead II:is composed of right arm which is made negative and the left leg, which is considered positive.

Lead III: is made up of the left arm which is designated as negative and the left leg which is considered positive.

The three standard leads form a triangle over the body and have a mathematical relationship to one another as described by Einthoven. The height or depth of the recordings in Lead I and Lead III equals the height (or) depth of the recordings in Lead II.

WILSON CENTRAL TERMINAL: The sum of the potentials from RA, LA, and LL is equal to Zero throughout the cardiac cycles with respect to any point at the body surface. Lead wires attached to electrodes on each limb re connected together through 5000-ohm resistors at a point. When this common point - Wilson's central terminal is attached to the negative pole of the ECG machine and an exploring electrode is connected to the positive pole the potential variations recorded will be those of the latter only. A lead taken by this method is called a unipolar lead. Actually the electrical potential of the central terminal is not truly zero because the right arm, left arm, and left leg are not equidistant from each other and from the heart, the body tissues vary in resistance and the heart and the extremities do not lie in exactly the same plane in the body. The potential of the central terminal has been said to average around $0.3 \mathrm{mv}$.

UNIPOLAR EXTREMITY LEADS: Unipolar extremity leads were initially recorded by a system in which the central terminal of Wilson constituted the indifferent electrode and the exploring electrode was one of the three of the limb electrodes. A lead is called indifferent because the potential existing at an infinite distance from these sources. Such leads were known as VR, VL and VF. At present unipolar extremity leads are obtained by disconnecting the input to the central terminal of Wilson from the extremity being explored. This result in a $1^{1 / 2}$ increase in the voltage. These augmented extremity leads are the ones currently used for clinical electrocardiography and are labeled AVR, AVL, AVF.

AVR: Augmented Voltage of the Right Arm. The right arm is the positive electrode in reference to the left arm and the left leg. This lead records the electrical activity of the heart from the direction of the right arm.

AVL: Augmented Voltage of the Left Arm. The left arm is the positive electrode in reference to the right arm and the left leg. This lead views the electrical activity of the heart from the direction of the left arm.

AVF: Augmented Voltage of the Left Foot. The left foot (or) the left leg is the positive electrode in reference to the left arm and the right arm. This lead sees the electrical activity of the heart from the direction of the bottom of the heart.

Unipolar Precordial Leads: The unipolar precordial ECG is obtained by placing exploring electrode on the classical six locations of the anterior and left portions of the chest. The central 
terminal is used as the indifferent electrode. Unipolar leads made by this method are prefixed by the letter $\mathrm{V}$ (which stands for voltage) followed by subscript numbers 1 to 6 , which indicate the corresponding chest sites. Precordial lead yields a positive deflection when facing positive charges and a negative deflection when facing negative charges. They do this according to what Wilson called the solid angle concept. A solid angle is an imaginary cone extending from the site in the chest throughout the heart. The precordial electrode is at its apex and its base is at the opposite epicardial surface. Precordial leads record the electrical activity from the regions of the heart over which they are placed as well as from distant regions. Thus if V2 is placed over the right ventricle part of the initial positive ventricular deflection reflects right ventricular activation with the corresponding electrical forces moving towards the electrode. Most portions of the terminal S wave represent activation of muscle other than the right ventricle reflective electric forces moving away from the electrode. Lead V2 is a right ventricular lead only because the electrode faces the right ventricle not because it records only the electrical activity of the right ventricle. The peak of the $r$ (or) $\mathrm{R}$ wave in this and all other precordial leads gives a rough estimate of the moment of arrival of excitation at the muscle underneath the electrode.

The following positions are used for placement of a suction cup lead on the chest in order to obtain the correct precordial lead placement.

V1- $\quad 4^{\text {th }}$ intercostals space immediately to the right of the sternum.

V2- $\quad 4^{\text {th }}$ intercostals space immediately to the left of the sternum.

V3- Directly between V2 and V4

V4- $\quad 5^{\text {th }}$ intercostal space - left mid clavicular line.

V5- $\quad 5^{\text {th }}$ intercostals space - left anterior axillary line.

V6- $\quad 5^{\text {th }}$ intercostals space - left mid axillary line.

These are precordial leads view the heart in the horizontal plane.

$\mathrm{V} 1$ and $\mathrm{V} 2$ are placed over the right ventricle.

V3 and V4 lie over the inter-ventricular spectrum.

V5 and V6 are placed over the left ventricle.

The data collected was included in the following format.

1. Sl.No.

2. Age

3. Sex

4. Standardization

5. Rate

6. Rhythm

7. Intervals-PR, QRS, QT

8. Axis: P axis, QRS axis

9. Position: Vertical, Intermediate, Horizontal

10. Rotation: Clockwise, Anti clockwise,

11 Segments: PR, ST

12. Other findings. 


\section{ORIGINAL ARTICLE}
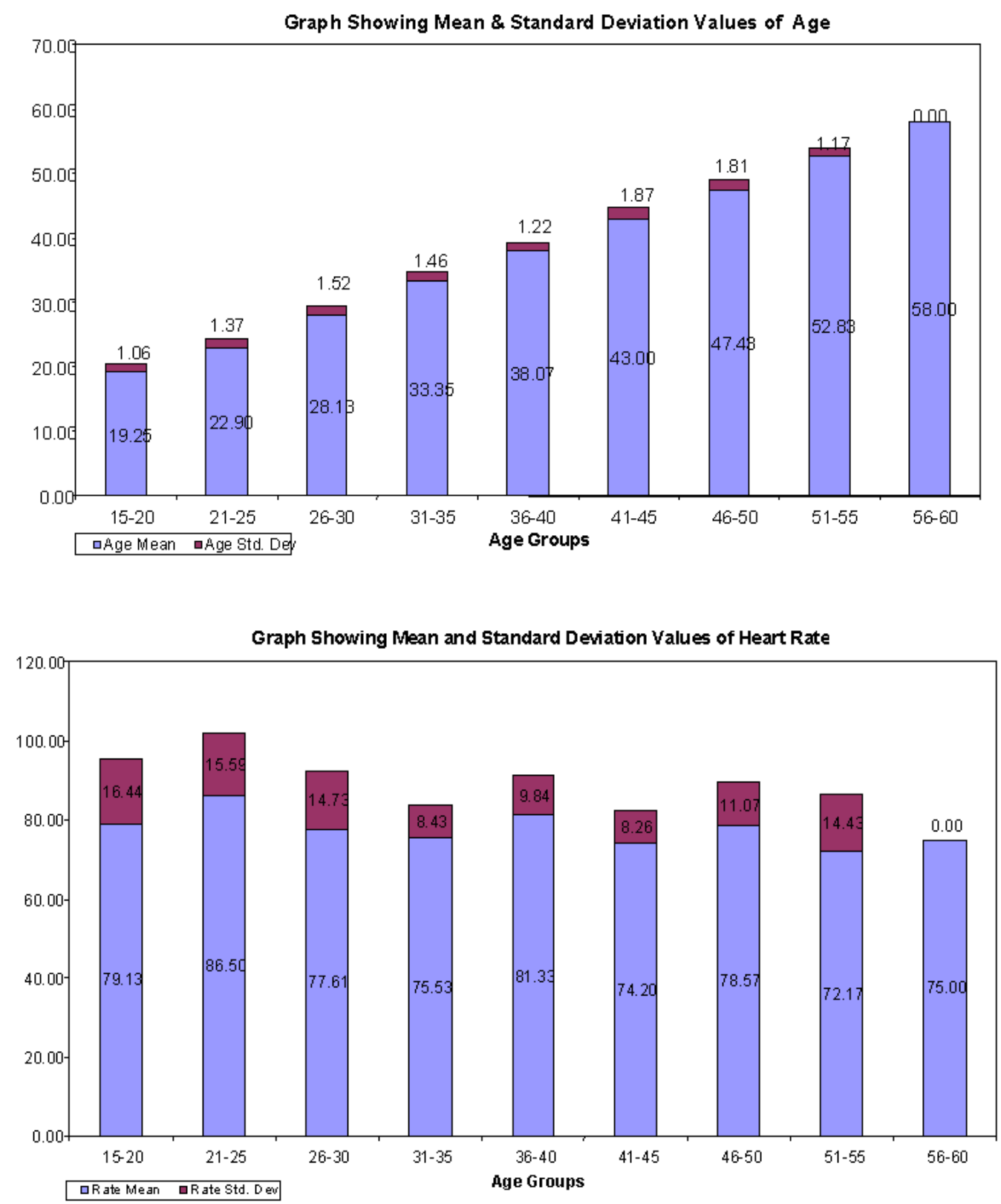


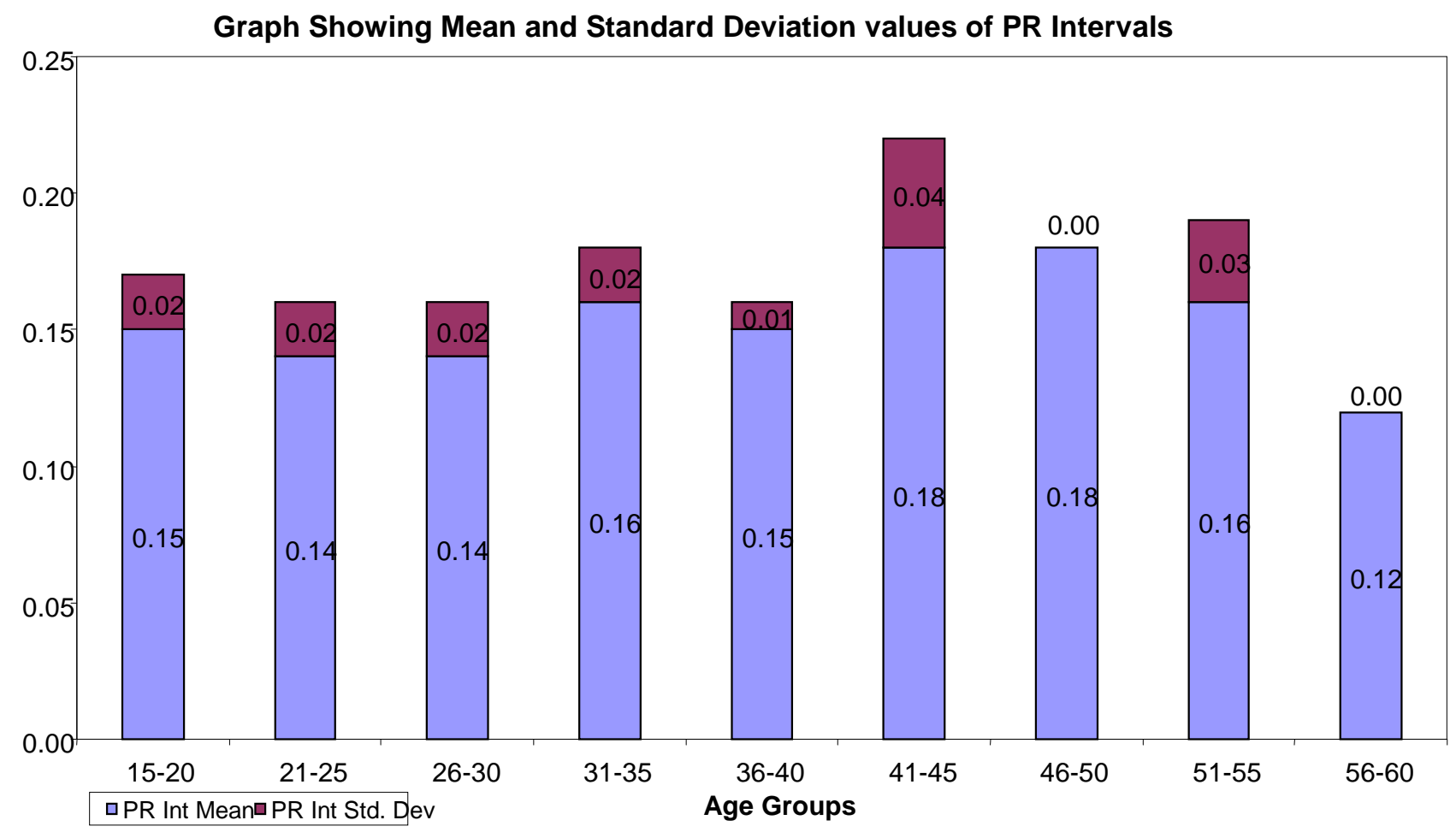

Graph Showing Mean and Standard Deviation Values of QRS Intervals

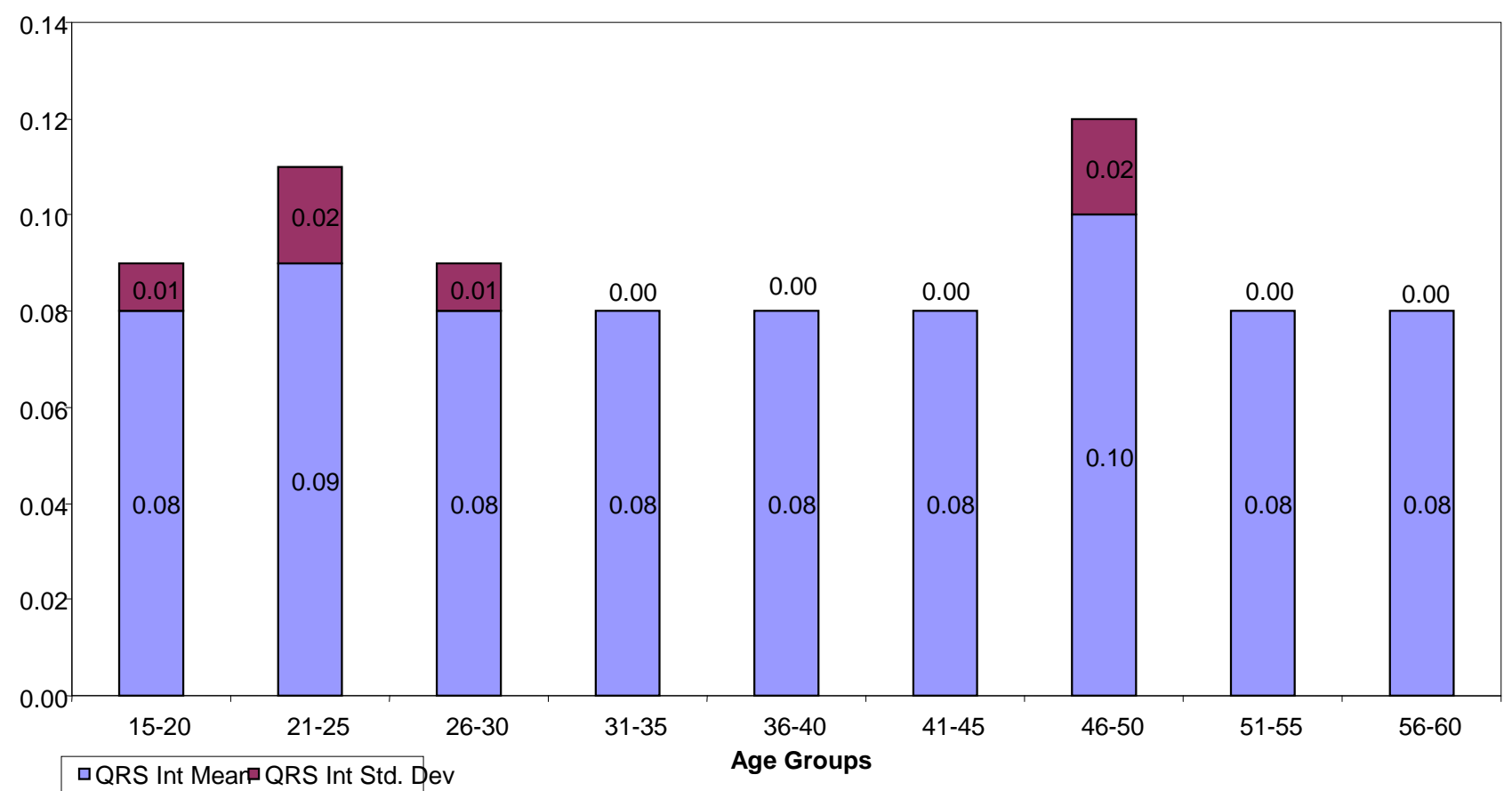




\section{Graph Showing Mean and standard Deviation values of QT Intervals}
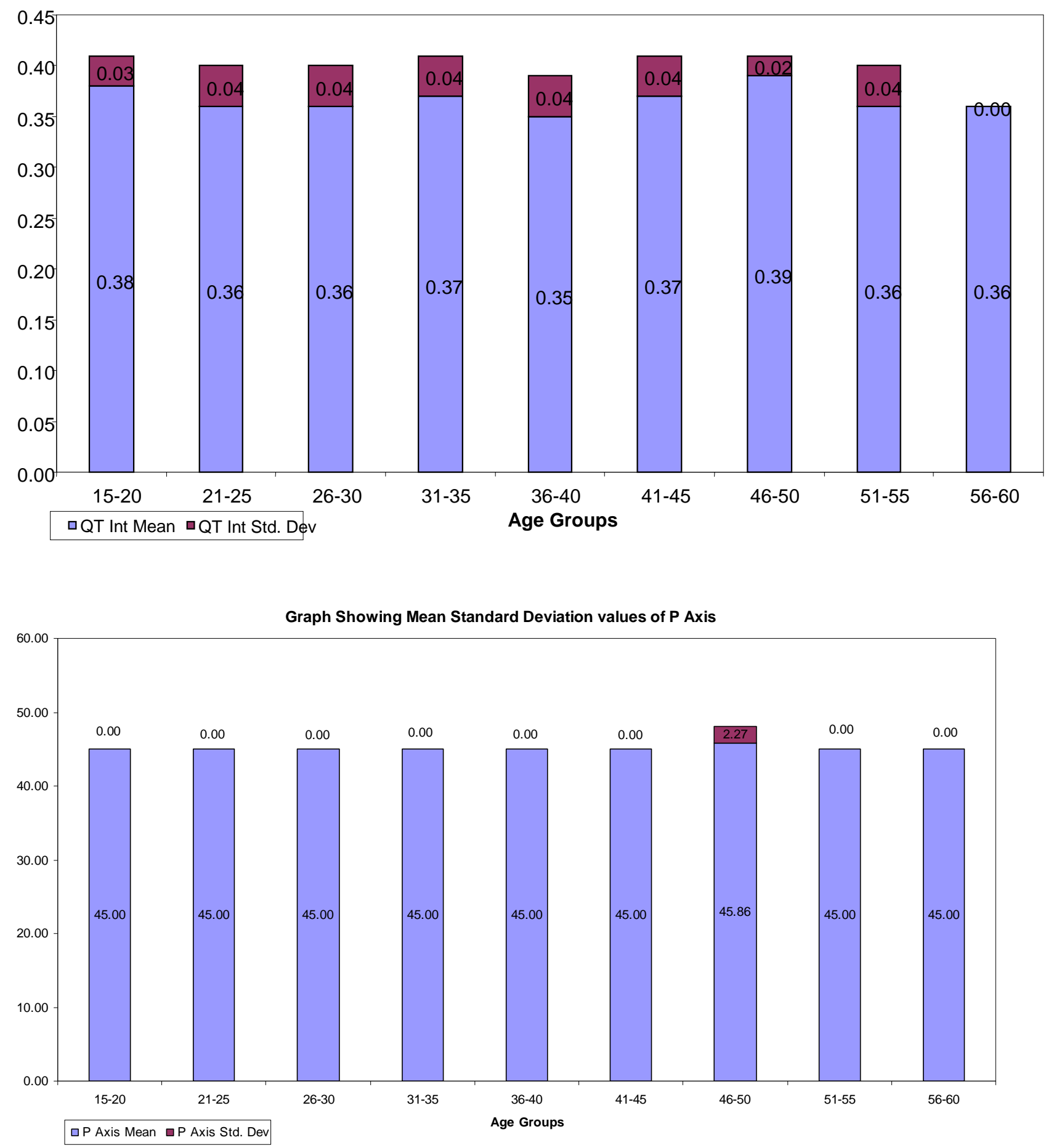


\section{ORIGINAL ARTICLE}
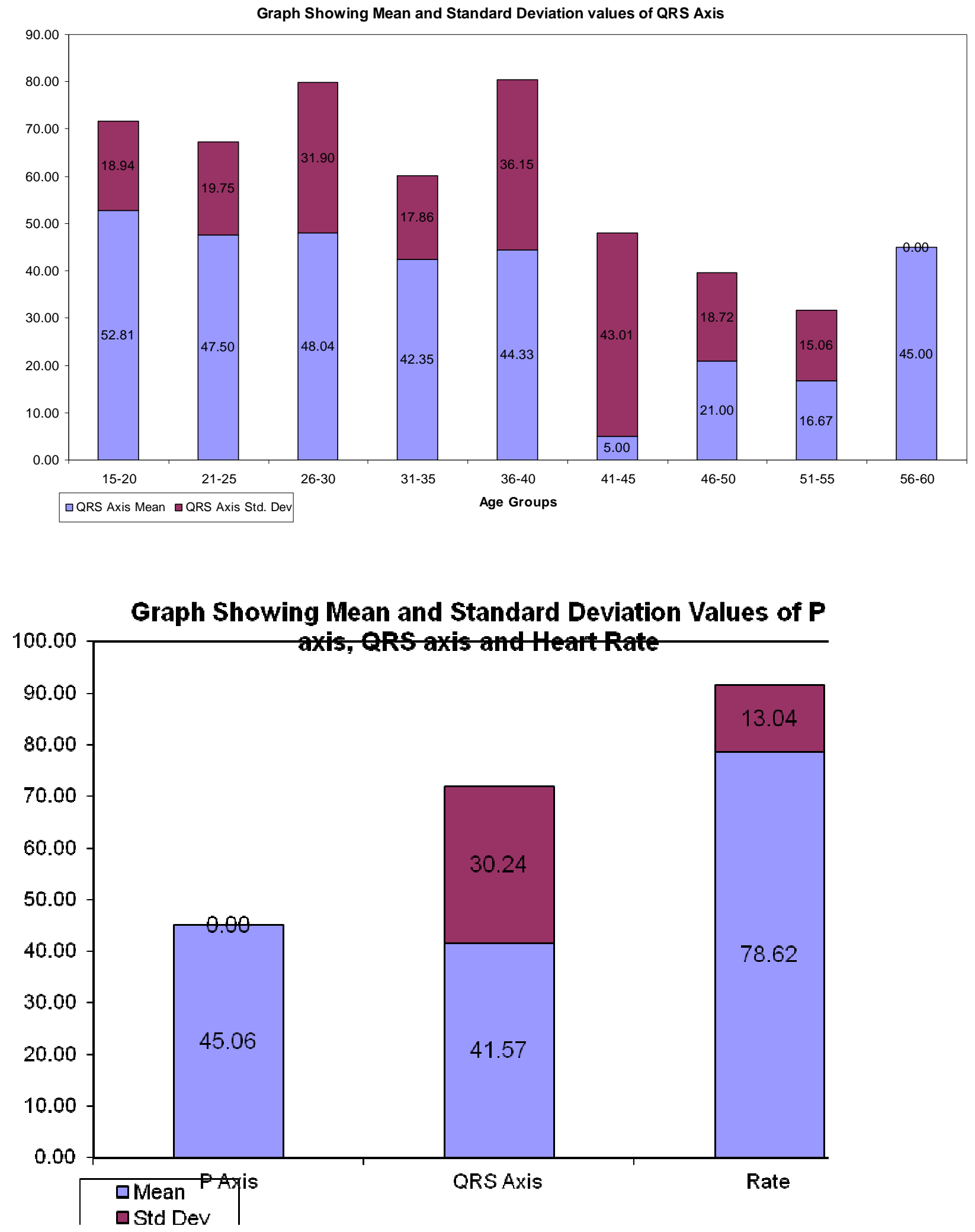


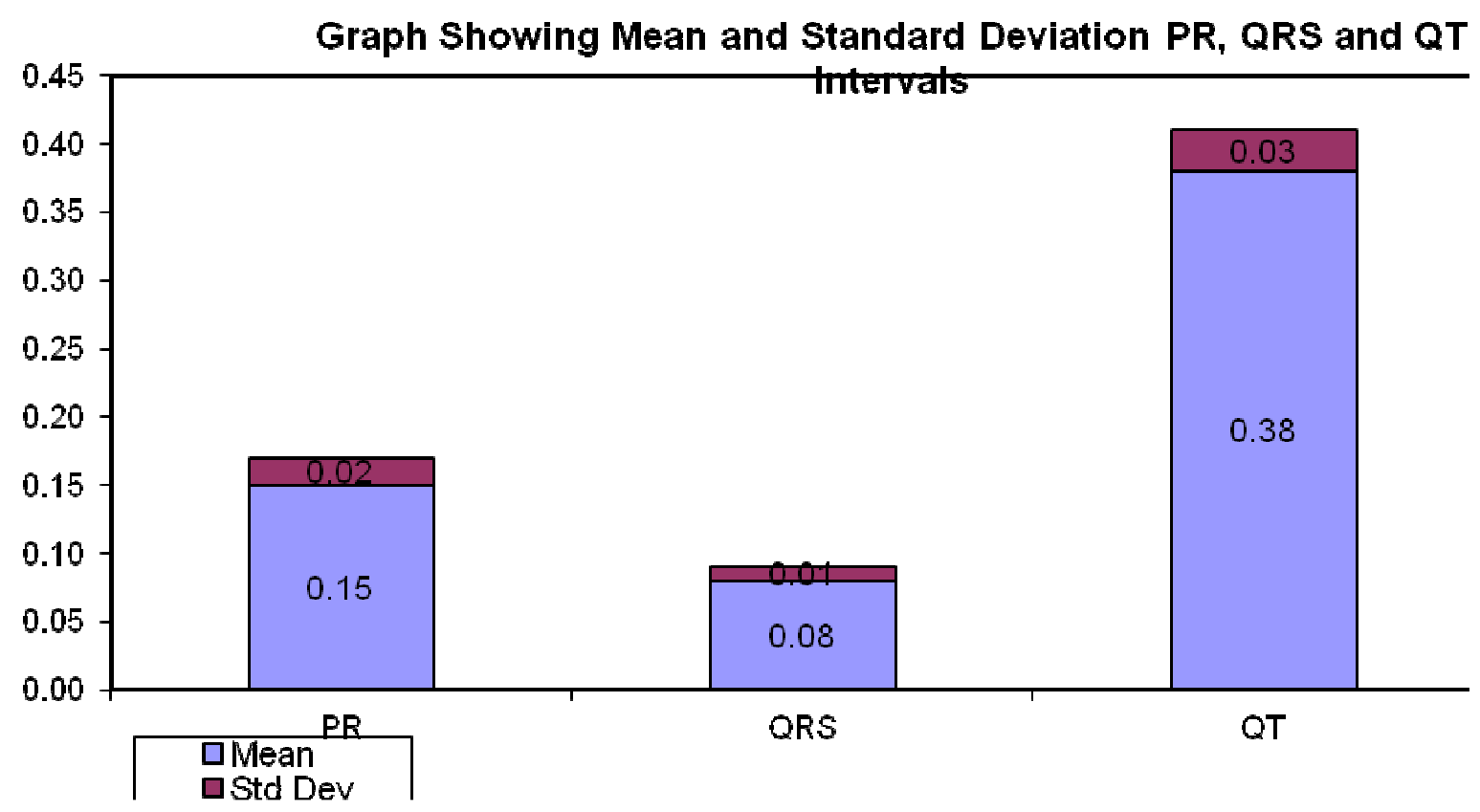

The present study includes 100 normal, healthy individuals out of which 72 were males, 28 were females. The lowest age is 16yrs and highest age is 58yrs. The heart rate varied between 46 and $112 \mathrm{beats} / \mathrm{min}$ taking 60 to $100 \mathrm{beats} / \mathrm{min}$ as normal range of heart rate. Below $60 \mathrm{beats} / \mathrm{min}$ was considered as Bradycardia and above 100beats/min as Tachycardia. Among the 100 studied 99 individuals showed normal sinus rhythm and one showed wandering pacemaker activity. Another significant finding about young individuals was higher heart rates but falling in normal range. There were three people with bradycardia, two females and one male all adults above $40 y r s(46,55,58)$.

Sinus Tachycardia was seen in 4 individuals and all were less than 40yrs [25, 20, 25, 28] 3 males and 1 female with tendency for tachycardia at lower age groups.

PR Interval: Varied between $0.12 \mathrm{sec}$ to $0.24 \mathrm{sec}$ (normal range- $0.12 \mathrm{sec}$ to 0.20 ). One person in whom PR interval is $0.24 \mathrm{sec}$ showed $1^{0}$ heart block. His age is 42 years and the same person is also having left axis deviation.

QRS duration: Varied between $0.08 \mathrm{sec}$ to $0.12 \mathrm{sec}$. Majority showing $0.08 \mathrm{sec}$ (normal range- 0.08 sec to $0.1 \mathrm{sec}$ ). Only one individual showing $0.12 \mathrm{sec}$, male aged, 46 years, presented with Right bundle branch block.

QT Interval ranged between 0.3 to 0.4 sec QRS axis: Only two patients showed (Case Nos.62, 80) no axis (Indeterminate axis). One with - 30 degrees and another with - 60 degrees one case (43) can be taken as LAD without any prolongation of QRS duration. The same patient also showed PR interval prolongation (i.e.,) first degree Heart Block. 
Position of the Heart: The electrical position of the heart, which may or may not be related to the anatomical position, is determined by just 2 leads AVL and AVF based on the above principle, the electrical position of the heart is determined as

\begin{tabular}{|c|c|c|c|}
\hline \multirow{3}{*}{$\begin{array}{c}\text { Direction of QRS } \\
\text { Complex }\end{array}$} & Lead AVL & Lead AVF & Position \\
\cline { 2 - 4 } & Dominantly + Ve & Dominantly +Ve & Intermediate \\
\cline { 2 - 4 } & Dominantly + Ve & Dominantly - Ve & Horizontal \\
\cline { 2 - 4 } & Dominantly - Ve & Dominantly +Ve & Vertical \\
\hline
\end{tabular}

Depending on the position of the heart, the complexes in AVL and AVF may be either positive or negative. Both can be positive and both can be negative. But in practice physician determines the axis of the heart in preference to its position, the former having more relevance than the latter in clinical practice.

Rotation of the Heart: Before discussing rotation of the heart, it is important to understand the orientation of the ECG leads with respect to the body .

Electrical rotation of the heart refers to a rotation in the horizontal place of the heart. The horizontal plane is represented by the chest leads $V_{1}-V_{6}$. A rotation in this plane is defined with reference to a view taken from below the diaphragm and is either clock wise or anticlockwise. There is however, a simple and practical way of determining the rotation of the heart. This depends on the zone of transition of the $\mathrm{R}$ and $\mathrm{S}$ waves in the mid precordial leads. If the zone of transition is seen in leads $V_{3}$ or $V_{4}$, then it is considered normal. If this zone shifts to $V_{1}$ or $V_{2}$ i.e., counter clockwise from $V_{2}, V_{4}$ and then the heart is rotated counter clockwise. Similarly if the zone of transition shifts to $V_{5}$ to $V_{6}$ then the heart is rotated clockwise.

\section{Anticlockwise}

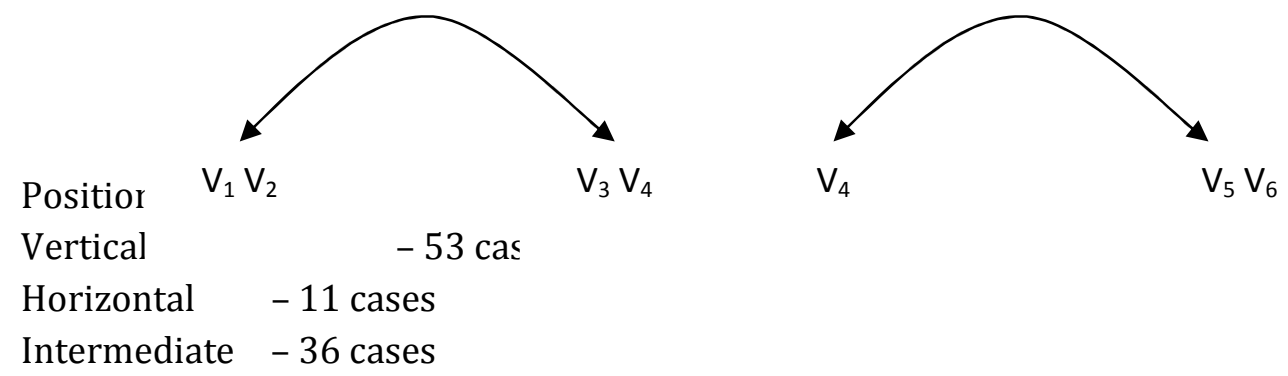

Rotation: Anticlockwise rotation is seen in nine patients, showed early appearance of $\mathrm{R}$ waves in the precordial leads.

ST Segment: ERS (Early Repolarization Syndrome): ST segment was iso electric in all except 5 individuals (case was 9, 19, 91, 97, 44) all less than 40 years. The segment elevation is preceded by a small spike, both put together qualifying for early repolarization syndrome.

Early Repolarization Syndrome of the heart ventricles occurs in population (23) over 13 years old rather evenly. In children it is encountered significantly more frequently than in adults in males 2 times more than in females. In, in-patients especially with gastrointestinal and nervous 
diseases and in patients with organic cardio vascular dis-orders the syndrome occurs less frequently.

Patients with thoracic pain and early repolarization were studied. E.R.S. was diagnosed on the basis of the presence of two criteria. ST elevation, upward concavity, notch or slur on QRS, asymmetrical $\mathrm{T}$ waves of large amplitude, $\mathrm{U}$ waves. Conclusion in this study was the electrocardiographic characteristics of ST elevation with upward concavity observed in precordial leads in patients with atypical precordial pain are important to establish a differential diagnosis, to be confirmed by previous electrocardiogram and cardiac enzymes.

DISCUSSION: Electrocardiogram is graphic representation of the electrical activity of the heart and its conduction through the pathways, which precedes the Mechanical contraction of the heart. The electrical activity can be recorded from various parts of the body, limbs and chest. All these are considered as indirect, semi direct leads.

The ECG is good indicator for the change in rate, rhythm, ischemia, electrolyte levels and in many other conditions.

The ECG in normal individuals though varies from individual to individual but lies in particular limits of normal range. The present study is aimed at knowing whether the ECG, shows any abnormalities in normal individuals if so, their frequency, type and significance. The design of the study was such that all the individuals with known diseases in Diabetes Mellitus, Hypertension, COPD and other common diseases and people, with habits like smoking, alcoholism which are known to alter the ECG were all excluded from the study. Infants were also not included because the ECG varies in infants. Adults with known history of congenital heart disease, IHD were also excluded from the study.

Certain abnormalities are known to occur is normal individuals. They indicate benign nature like PVC's (Premature Ventricular Contraction) or sometimes a serious disorder which is silent or in some case it could be congenital.

The present study which included both, males and females showed the following abnormalities (knowing about the abnormalities in normal looking people is very important, in treating individuals).

Out of 100 patients 15 showed deviation from the normal pattern. 85 persons ECG were well within normal limits i.e. The waves, intervals, positions, rate, rhythm and axis. Sinus Tachycardia was seen in 4 cases (case No.18, 37, 68, 71) graph No.1 among these 4 individuals.

3 males and one female all were below 40 years, indicating it is common in younger individuals and with male predominance. Whereas sinus bradycardia was seen is 3 people, all above 40 years of age graph No.1 (Case No.10, 30,42) 2 females and 1 male.

Right Bundle Branch Block: R.B.B.B. which was seen in one (Case No.48) individual without any other evidence of ischaemia. This can be seen is normal individuals.

Premature ventricular Contractions: In the present study PVCs were seen is one individual . It is known that they can occur in normal individuals. It is important in making out the abnormality and ruling out the organic disease in the heart and important in treating them. For example PVCs in normal individuals are harmless. P.V.Cs with acute MI are associated with arrhythmias. 
First Degree Heart Block: Persons with PR intervals more than $0.20 \mathrm{sec}$ were considered having first degree heart block. If they present with low heart rate may not have significance, but with higher heart rates definitely indicates an abnormality of the conducting system. In this study it is seen in one Case No.40, Graph No.2.

Left Axis Deviation: It is seen in one individual, Graph No.3, No.43.

Early Repolarization Syndrome: This is characterized by ST elevation more than $3 \mathrm{~mm}$ in precordial leads with upward concavity preceded by a spike. 5 individuals showed this abnormality all are males and less than 40 years. This shows that it is due to repolarization of certain areas of the heart early in the cardiac cycle. The practical importance is that the ST elevation should be distinguished from that of ST elevation of ischaemic heart disease especially acute MI. It is also observed that complexes return to normal shape with higher heart rate, example after exercise.

\section{Wandering Pacemaker:}

Seen is one individual No.44, Graph No.2.

List of abnormalities in the present study.

$\begin{array}{llll}\text { 1. } & \text { Sinus Tachycardia } & - & 4 \\ \text { 2. } & \text { Sinus Bradycardia } & - & 3 \\ \text { 3. } & \text { PVC's } & - & 1 \\ \text { 4. } & \text { Wandering pacemaker } & - & 1 \\ \text { 5. } & \text { RBBB } & - & 1 \\ \text { 6. } & \text { LAD } & - & 1 \\ \text { 7. } & 1^{0} \text { Heart Block } & - & 1 \\ \text { 8. } & \text { ERS } & - & 5\end{array}$

CONCLUSION : ECG varies from individual to individual even in normals. Majority of the ECG's in normal individuals shows a pattern, which falls within normal range.

A considerable percentage of individuals deviate from the normal pattern. The frequently observed abnormalities are Tachycardia, Bradycardia and ERS.

Less frequently seen abnormalities are LAD, $1^{0}$ heart block PVC's, wandering pacemaker, RBBB.

So understanding normal ECG and deviations in normal individuals is very important to interpret the diseased states, treating them, and giving the prognosis.

\section{BIBLIOGRAPHY}

1. Gerard Tortora \& Sandra Reynolds Grabonslei, Principles of Anatomy and Physiology.

2. Goldmann; (ECG) Clinical Electrocardiography).

3. Dale Davis; ECG Interpretation,.

4. Fye $\mathrm{W}_{\mathrm{B}}$. A History of the origin, and impact of electrocardiography, Am Journal of Cardiol 1994; 73; 937-949.

5. Burchell HB. A centennial note on Waller and the first human electrocardiogram. Am Journal of Cardiol 1997; 59; 979-983. 
6. Burnett J. The origins of the electrocardiograph as a clinical instrument. Medical History. Supplement 5: 1985, 53-76, Published as a monograph. The emergence of modern cardiology. Bynum WF, Lawrence C, Nutton V, eds. Wellcome Institute for the History of Medicine 1985.

7. Hewbet - Packard - History and Mission.

8. The normal resting electrocardiogram - Augustin Castellanos / Kenneth M. Kessler / Robert J. Myerburg.

9. Harrison's Principles of Internal Medicine.

10. An Introduction Electrocardiography - Leo Schamroth.

11. Understanding Electrocardiography - Pradip J. Mehta.

12. Atterhog, J. H and Loogna E.P; R interval in relation to hart rate during exercise and the influence of posture and autonomic tone J. Electrocardiol 1977; 10, 331.

13. Lister J. Wetal; Atrio Ventricular conduction in man; effect of rate, exercise, isoproterenol and atropine on the P-R- Interval AM. J. Cardiol 1965; 16, 516.

14. Manning, G.W. Electrocardiography in the selection of Royal Canadian Air Force air review circulation 1954; 10, 401.

15. Johnson, R.L. et al., Electrocardiographic findings in 67, 375 asymptomatic individuals part VII, A - V. Block AM J. Cardiol 1960: 6, 153.

16. Manning J.W. and Sears G.A. Postural Heart Block Am. J. Cardiol - 1962; 9, 558.

17. bachman S et al., Effect of aging on the Electrocardiogram Am J. Cardiol, 1981; 48, 513.

18. Perloff J. K et al., Left axis deviation a reassessment circulation 1979; 60, 12. The electrical QRS axis may be shifted to the right in normals, during inspiration, dextrocardia. The electrical QRS axis is shifted to the left in normal individuals, expiration, Pregnancy.

19. Marriotte; Practical Electrocardiography.

20. Johnson R.L. Averill K.H. and Lamb L.F. 1960. Electrocardiographic Findings in 67, 375. Asymtomatic individuals part VI, Right bundle branch block Am. J. Cardiol, 143.

21. Ediekew J. Elevation the RS-T segments apparent or real in the right precordial leads as a probable normal variant. Am Heart J 1954; 48, 331.

22. Goldman, M.J. RS-T segment mid and left precordial leads as a normal variant Am Heart J $1953 ; 46,817$.

23. Prevalent of Early Repolarization Syndrome. Department of Internal Diseases Krasnoirsk Medical Institute.

24. Vacanti L.J. Thoracic pain and ERS at the Cardiological emergency Unit. Sancor-pronto Soeorro do Carac ao de Santos.

25. Diurnal Pattern of QTC interval possible relation to circadian triggers of cardiovascular events. Reingold ECG, Center. Department of Medicine Northwestern University Medical School, Chigago, Illinois 60611 - 3008 USA.

26. Spectral components of short term R.R. - Interval variability in healthy subjects and effects of risk factors. Department of Cardiology, Skejby University Hospital, Arhus, Denmark.

27. Signal averaged ECG shows a heart rate dependent diurnal variation healthy subject. Department of Laboratory Medicine, Oita Medical University, Japan.

28. Heart rate variability is healthy subjects in related to age and gender. Department of Clinical Physiology, Soder Hospital, Karolinska Institute, Stockholm, Sweden. 


\section{ORIGINAL ARTICLE}

29. Circadian variation of QT internal dispersion: Correlation with heart rate variability. Department of Laboratory Medicine, Oita Medical University, Japan.

30. ECG changes in asymptomatic healthy males. Indian Journal of Physiology Pharmacology, 1994.

31. Prevalence of asymptomatic electrocardiographic abnormalities in a rural population. Department of Medicine, Monilek Hospital and Research Center, Jawhar Nagar, Jaipur, India.

32. Heart rate and heart rate variability in normal young adults. Hospital Lariboisiere, Paris, France.

33. A study on frequency characteristics of fitter for detecting high frequency components of QRS complexes and its applications to the Intra Ventricular conducting systems. Second department of Internal medicine St. Marianna University, School of Medicine.

34. Electrocardiographic changes in patients with chronic anemia. Institute of Urology and Nephrology Clinical Centre of Serbir, Belgrade.

35. Electrocardiographic monitoring in healthy young adult out patients mandatory or optional. Department of Anesthesia Vancouver Hospital and Health Sciences Centre, University of British Colombia, Canada.

36. Eleven year old made with high voltage electrical injury and premature ventricular contractions. Department of Emergency Medicine, Olive View - UCLA Medical Centre, USA. 


\section{ORIGINAL ARTICLE}

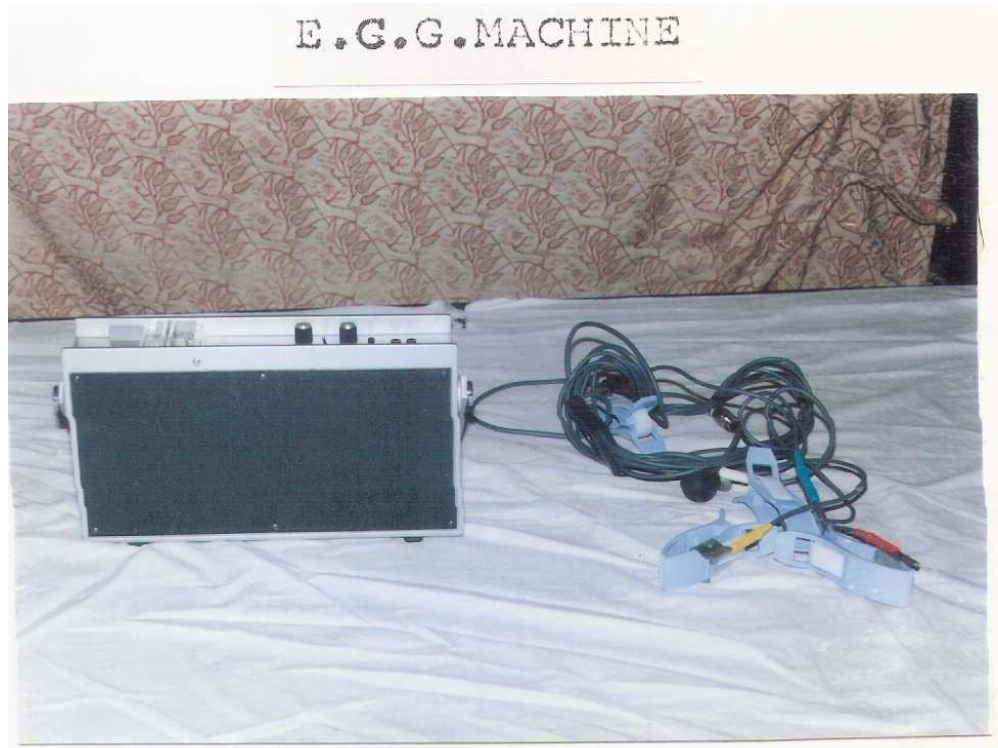

RECORDING OF E.C.G.

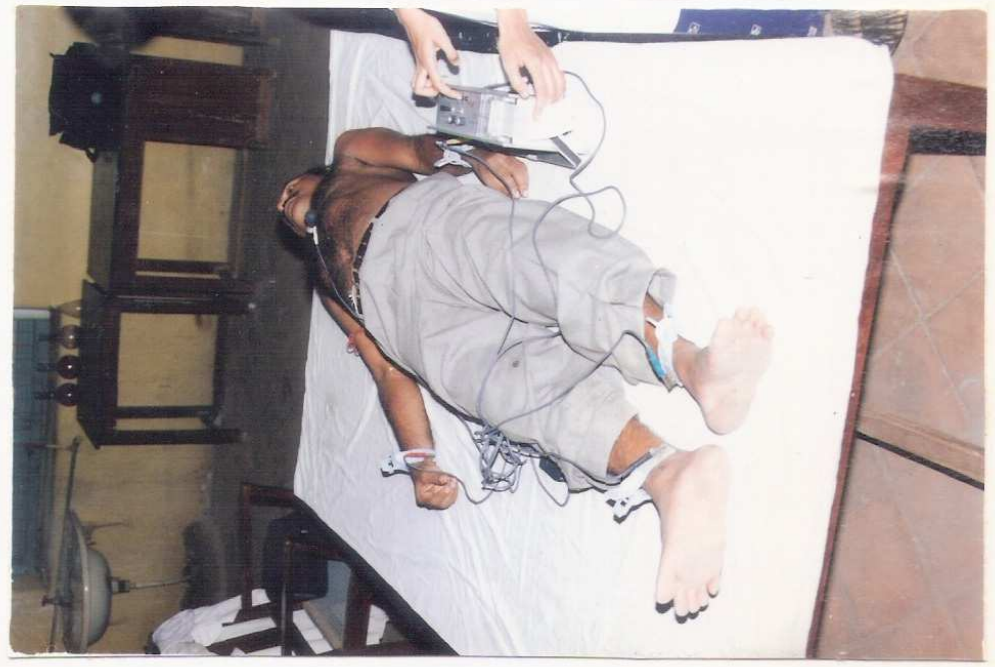

